\title{
Synchronous Sampling and Clock Recovery of Internal Oscillators for Side Channel Analysis and Fault Injection
}

\author{
Colin O'Flynn · Zhizhang (David) Chen
}

\begin{abstract}
Measuring power consumption for side channel analysis typically uses an oscilloscope, which measures the data relative to an internal sample clock. By synchronizing the sampling clock to the clock of the target device, the sample rate requirements are considerably relaxed; the attack will succeed with a much lower sample rate.

This work characterizes the performance of a synchronous sampling system attacking a modern microcontroller running a software AES implementation. This attack is characterized under four conditions: with a stable crystal-oscillator based clock, with a clock that is randomly varied between $3.9 \mathrm{MHz}-13 \mathrm{MHz}$, with an internal oscillator that is randomly varied between 7.2 MHz-8.1 MHz, and with an internal oscillator that has slight random variation due to natural 'drift' in the oscillator.
\end{abstract}

Traces captured with the synchronous sampling technique can be processed with a standard Differential Power Analysis (DPA) style attack in all four cases, whereas when an oscilloscope is used only the stable oscillator setup is successful. This work also develops the hardware to recover the internal clock of a device which does not have an externally available clock. It is possible to implement this scheme in software only, allowing it to work with existing oscilloscope-based test environments.

Performing the recovery in hardware allows the use of fault injection with excellent temporal stability relative to a sensitive event. This is demonstrated with a power glitch inserted into a microcontroller, where the glitch is triggered based on a signature in the measured power consumption.

Dalhousie University, Halifax, Canada

E-mail: \{coflynn, z.chen\}@dal.ca

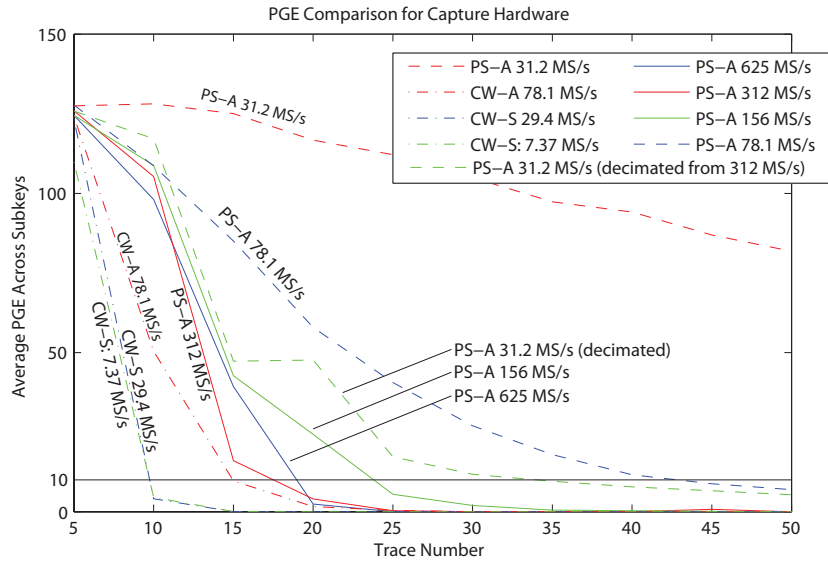

Fig. 1 PS-A means the PicoScope 6403D sampling at the given sample rate, asynchronous to the device clock. CW-A means the ChipWhisperer in asynchronous mode at the given sample rate, and $\mathrm{CW}-\mathrm{S}$ means the ChipWhisperer in synchronous mode.

Keywords side-channel analysis, acquisition, synchronization, DPA

\section{Introduction}

By measuring the power consumed by a digital device on each clock cycle, it is possible to infer something about the data being processed by this device. This was demonstrated as a method of breaking cryptographic cores using Differential Power Analysis (DPA)[7]. Such measurements are typically done with standard oscilloscopes, which depending on the attack algorithm and device under attack may range from simple low-cost oscilloscopes to high-end specialist oscilloscopes. But if the underlying objective is to measure data on the clock edges of the system clock, sampling at the clock rate of the system is sufficient, provided such samples occur at 
the correct moment (i.e. on the clock edge). This sampling technique is called synchronous sampling, where the sample clock is synchronized to the device clock. The application of this to side-channel analysis was first described in Section 5.2 of [10]. A demonstration of this technique to attack the SASEBO-GII board is given in [12], where sampling at $96 \mathrm{MS} / \mathrm{s}$ synchronously achieves similar results to $2 \mathrm{GS} / \mathrm{s}$ asynchronously.

For this to be successful, the previous work assumed that the system clock was readily available. For many systems this will be the case - an external oscillator or clock drives the digital logic, and it is trivial to tap into this clock. But many devices rely instead on an internal oscillator; there is no clock signal available for synchronous sampling. In addition devices may purposely vary the frequency of the internal oscillator in an attempt to stop power traces from synchronizing in the time domain, requiring the attacker to resynchronize the traces after capture. The varying clock countermeasure is assumed to be difficult to reverse in most instances. For example it is claimed in [21] that varying the clock frequency "makes time correlation, a very important step in power analysis attacks, impossible."

If the data was capture asynchronously (i.e. with a normal oscilloscope) with sufficient sample rate, it's possible to compensate for the varying clock frequency via post-processing. This is of little use for attacks requiring real-time information: a trigger matching an analog pattern in the power data, or the injection of glitches timed to specific events requires real-time knowledge of the device clock.

This work addresses the problem of recovering the clock from a device under test for both side-channel analysis and fault injection. First, an introduction to the reference platform being used is given, along with a comparison of the synchronous sampling technique to standard asynchronous sampling on this platform.

The platform is then changed to use an internal oscillator which actively varies the frequency during cryptographic operations. Attacks using standard asynchronous oscilloscopes without preprocessing, with preprocessing, and synchronous sampling are all compared.

Finally a method of performing clock recovery, and using that clock for synchronous sampling is demonstrated. The clock recovery method can be seen as a hardware implementation of the software preprocessing technique. The use of Sum-of-Absolute Difference (SAD) triggers to detect specific events in the system is demonstrated, and finally the injection of glitches is performed on the target while the operating frequency varies.

\section{Experimental Platform}

The device under test (DUT) is an Atmel AtMega48A microcontroller in 28-pin DIP. This device was selected due to several clocking features: it can use an internal or external clock source, the internal oscillator can be adjusted by firmware running on the microcontroller during operation, and the internal clock can be output onto an I/O pin. The differential voltage is measured across a shunt inserted into the VCC pin of the microcontroller. For asynchronous sampling a PicoScope 6403D oscilloscope is used, and for synchronous sampling the ChipWhisperer is used. Full details of the capture hardware and software are available in [13] and at the ChipWhisperer wiki ${ }^{1}$. See Fig. 18 for a photo of the test setup.

The 'A' suffix for the AtMega48A indicates it is using a recent fabrication process; the older AtMega48P by comparison is made with a larger $(0.35 \mu \mathrm{m})$ process. The AtMega48P draws more power, and thus would be expected to give a stronger signal across the resistive shunt used to measure current. The AtMega48A thus reflects a reasonable platform which can be compared against any recent digital $\mathrm{IC}^{2}$.

The crypto module under attack is a $\mathrm{C}$ implementation of the AES-128 algorithm. The specific C implementation chosen was 'AES in C' available from avrcryptolib $^{3}$. The attack algorithm is a standard Correlation Power Analysis (CPA) attack[3].

\subsection{Comparison of Sampling Platforms}

While the ChipWhisperer is capable of using both asynchronous and synchronous sampling, it is limited to a maximum sample rate of $100 \mathrm{MS} / \mathrm{s}$. For comparison of higher speed asynchronous captures, a PicoScope 6403D is used for asynchronous sampling, which can achieve up to $5 \mathrm{GS} / \mathrm{s}$.

Fig. 1 shows a comparison between the different oscilloscopes and sampling types. For this figure an external $7.37 \mathrm{MHz}$ crystal oscillator was used as a clock source. Results in this paper will be an average of the partial guessing entropy (PGE) of all subkeys, and where space permits the PGE of each individual subkey is graphed. The reader is referred to Section 2.2 if they are unfamiliar with the PGE metric.

With the PicoScope 6403D (the PS-A data from Fig. 1), it is noted that increasing sample rates have

\footnotetext{
1 www.chipwhisperer.com

2 The feature size of this specific device is unknown, but based on similar devices is assumed to be within the $0.12 \mu \mathrm{m}$ $-0.18 \mu \mathrm{m}$ range

3 http://avrcryptolib.das-labor.org
} 
improved attack performance initially, but beyond a certain point almost no improvement occurs. For this attack setup there is minimal change from $156 \mathrm{MS} / \mathrm{s}$ to $625 \mathrm{MS} / \mathrm{s}$, and in particular the results of $312 \mathrm{MS} / \mathrm{s}$ and $625 \mathrm{MS} / \mathrm{s}$ are almost indistinguishable.

In the introduction of synchronous sampling, it has been previously claimed the main issue is the random jitter between the trigger event and the first sample occurring that causes the poor performance at lower sample rates in asynchronous systems [10]. We would thus expect a system using a fast sample rate for capture (i.e. so the jitter between the trigger and first sample is minimized), but decimated to a lower sample rate, to have better performance than simply selecting a lower sample rate.

In Fig. 1 the line labelled $P S-A$ 31.2 $M S / s$ (decimated from $312 \mathrm{MS} / \mathrm{s}$ ) is captured in such a fashion. The $312 \mathrm{MS} / \mathrm{s}$ data is decimated to $31.2 \mathrm{MS} / \mathrm{s}$ by selecting every $10^{\text {th }}$ data point and writing them to a new trace file, which the attack is run against. Note that the performance is considerably better than the capture which originally occurred at $31.2 \mathrm{MS} / \mathrm{s}$. No anti-aliasing or other filter has been used in the decimation process. Certain oscilloscopes contain a feature to capture at a high sample rate, and perform such downsampling the PicoScope 6403D for example provides this option, although this feature is not used in this work.

The performance of the ChipWhisperer hardware at $78.1 \mathrm{MS} / \mathrm{s}$ in asynchronous mode shows considerably better performance than the PicoScope 6403D at 78.1 MS/s. It is assumed the built-in Low Noise Amplifier (LNA) in the front-end is resulting in less noise, compared to the PicoScope 6403D which has a more general-purpose front-end.

Finally, note the ChipWhisperer hardware in synchronous mode results in further improvement in performance, despite the considerably reduced sample rates. In synchronous mode the device must sample at a multiple of the $7.37 \mathrm{MHz}$ clock, so sampling is done at $7.37 \mathrm{MS} / \mathrm{s}$ and 29.4 MS/s. Both of these results are almost indistinguishable on the graph, indicating that on this particular hardware using a single sample per clock is sufficient.

\subsection{Meaning of PGE}

The 'guessing entropy' can be defined as the "average number of successive guesses required with an optimum strategy to determine the true value of a random variable $X "[9]$. In this paper the 'optimum strategy' is to take the output of the attack, and rank the possible values of the subkey from most to least likely.

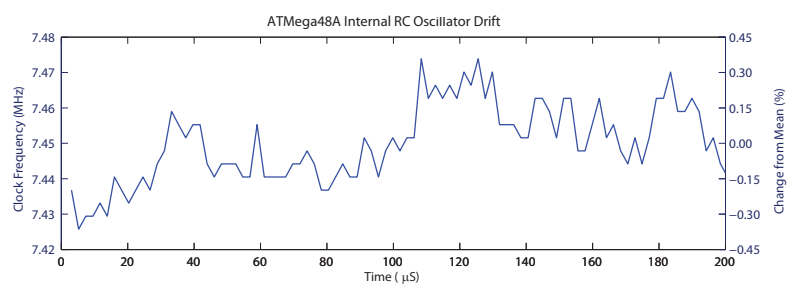

Fig. 2 Atmel AtMega48A internal clock drift during a sidechannel attack.

The 'partial' refers to the fact that we are finding the guessing entropy on each subkey. This gives us a PGE for each of the 16 subkeys $^{4}$. A PGE of 0 indicates the subkey is perfectly known, a PGE of 10 indicates that 10 guesses were [incorrectly] ranked higher than the correct guess.

The PGE for each subkey is calculated when the attack algorithm has access to $1,2, \cdots, N$ traces. We record the number of traces when the maximum PGE across all subkeys falls below 10. To improve consistency the PGE for each subkey is averaged over several attacks (trials).

\section{Varying Clock Frequency}

When an attacker is recording the power traces, ideally each trace would be perfectly synchronized with each other. That is to say that each time instance across all traces corresponds to the same instruction occurring on the DUT. In real systems, traces may not be perfectly synchronized. This could come from jitter in the trigger signal, unintended non-linear code flow such as interrupts on the DUT, or countermeasures such as instruction shuffling or random delay insertion. A discussion of algorithms and their performance for resynchronizing is compared in [5]. For all these events the clock is operating at a constant frequency.

Another class of synchronization aims to compensate for the clock frequency of the device varying (called varying clock or $\mathrm{VC}$ ), either due to countermeasures or simply due to the oscillator drift. For an example of the natural variation see Fig. 2, which was measured the short-term drift of the internal oscillator on the experimental platform used here. This small amount of variance was enough to prevent the same CPA attack from being successful with over 2500 traces $^{5}$, when with a stable crystal oscillator it was successful in only 30 traces. Algorithms which aim to reverse the $\mathrm{VC}$ are given in $[20,6,15,18]$.

\footnotetext{
4 This paper is always using AES-128

5 After 2500 traces the average PGE was 40, and only 4 of the 16 bytes had a stable $\mathrm{PGE}<5$
} 
When a large number of points are required per trace or a large offset from the trigger to the points of interest exist, even the short-term drift differences between the oscillator in the DUT and the oscillator in the oscilloscope may result in desynchronized traces.

With synchronous sampling, variations in clock frequency will naturally be eliminated from the data source. Each sample no longer corresponds to a time instant, but instead to a clock transition. Synchronization may be required for reasons previously discussed such as trigger jitter or countermeasures, but is not needed to compensate for the clock frequency changing.

\subsection{Synchronous Sampling of Varying Clock}

As a demonstration of synchronous sampling under VC conditions the AtMega48A target was designed to randomly vary the internal clock frequency before calling the AES encryption routines, and a side-channel attack was mounted. For this initial test the CLKOUT fuse was programmed to output the internal clock onto an IO pin, and the sampling is done synchronous to this clock.

\subsubsection{Internal Oscillator Adjustment Range}

The AtMega48A datasheet guarantees the oscillator can be calibrated between $7.3 \mathrm{MHz}-8.1 \mathrm{MHz}$, but the actual range is much larger - the specific part used here had a range of $3.95 \mathrm{MHz}-13.0 \mathrm{MHz}$. This test is operating the device outside of guaranteed operating range; commercial products would be advised to only use the adjustment over a smaller range. The time required to switch from the two possible extremes of the randomly selected frequencies, $3.9 \mathrm{MHz}$ to $13 \mathrm{MHz}$, is shown in Fig. 3. The datasheet specifies a maximum change of $2 \%$ clock cycle period between cycles for an external clock; it is not clear if this rapidly changing internal oscillator would also be subject to these considerations[1]. For this reason a number of NOP instructs are inserted before beginning further processing after changing the OSCCAL register.

Table 1 For the ATMega48A, several different clocking options are used. Two of them purposely vary the frequency of the internal oscillator, one uses the internal oscillator without adjustment, and one uses the standard crystal oscillator.

\begin{tabular}{lccl}
\hline Name & Range $(\mathrm{MHz})$ & Mean $(\mathrm{MHz})$ & Std-Dev \\
\hline Extended & $3.945-12.96$ & 7.210 & $2.190 \mathrm{MHz}$ \\
Narrow & $7.247-8.110$ & 7.663 & $287.5 \mathrm{kHz}$ \\
Drift & $7.315-7.413$ & 7.358 & $11.78 \mathrm{kHz}$ \\
Crystal & $7.373-7.373$ & 7.373 & $5.469 \mathrm{~Hz}$ \\
\hline
\end{tabular}

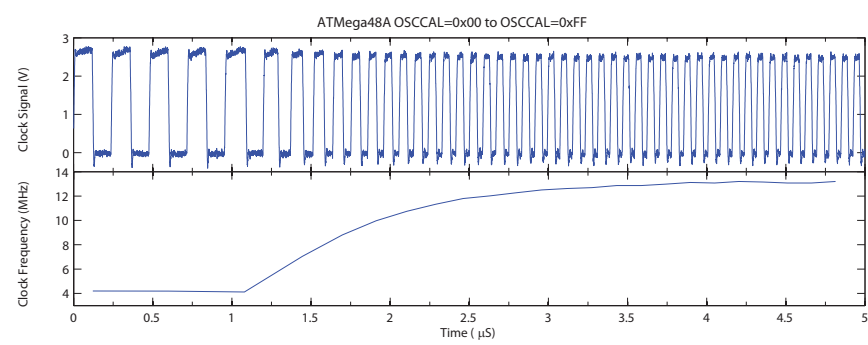

Fig. 3 Atmel AtMega48A internal clock frequency change as OSCCAL changes from 0 to 255 .

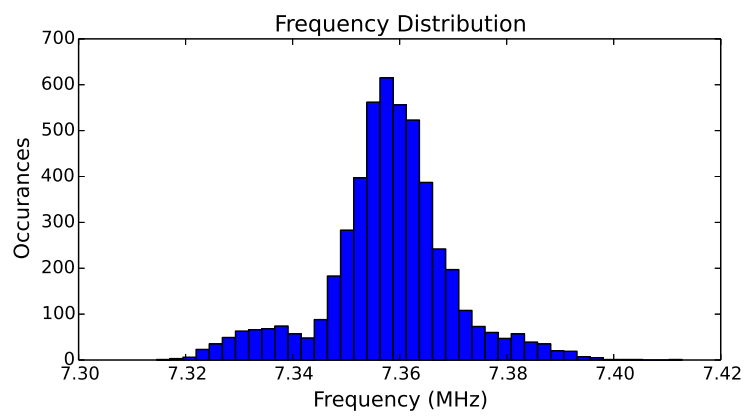

Fig. 4 The histogram of the operating frequency for the 'drift' range. The distribution appears to approximately follow the Normal distribution.

\subsubsection{Internal Oscillator Ranges Used}

In this course of this paper, three 'ranges' are used for adjustment of the internal oscillator. The first is the extended range, as mentioned spans from $3.9 \mathrm{MHz}-$ $13 \mathrm{MHz}$. A smaller narrow range is also used, which limits the adjustment to a level consistent with the datasheet. Finally the drift range is also explored, which reflects the natural random variations due to the nature of the internal oscillator in this device. Detailed information about each of those ranges is presented in Table 1 . To validate the frequency measurement system, the crystal range is also included, where a crystal oscillator is used to maintain a perfect clock reference.

In Fig. 4, the histogram of operating frequency during the requested encryptions is shown for the 'drift' range. This appears to follow the normal distribution, as would be expected by a process resulting from random noise. In Fig. 5 the histogram is shown for the 'extended' operating range. The value written to the adjustment register (OSCCAL) is uniformly random in the range $[0,255]$. The AtMega48A splits the OSCCAL register into two over-lapping frequency ranges. Furthermore it does not have linear mapping from the OSCCAL register to operating frequency, resulting in a non-standard distribution[1]. 


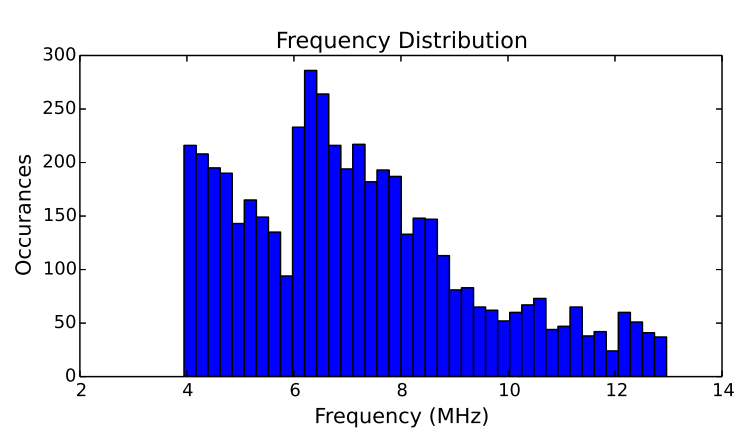

Fig. 5 The histogram of the operating frequency for the 'extended' range. The non-linear mapping of the control register to operating frequency, which is also split into two overlapping ranges, results in a non-standard distribution.

\subsection{Preprocessing of Traces}

The power consumption of a digital device is dependent on the frequency of operation, and this follows a linear relationship. For the ATMega48A at 3.3V, the power consumption when moving from $3.9 \mathrm{MHz}$ to $12 \mathrm{MHz}$ goes from $1.7 \mathrm{~mA}$ to $3.1 \mathrm{~mA}[1]$. While the power traces will line up in the time domain with synchronous sampling, they will require scaling in order to allow comparison of the same point across multiple traces. In [15] it is suggested to add an adjustment factor based on the measured frequency of operation, as in (1). Here $T_{p, n}$ is a single point at index $p$ in trace $n, C$ is a scaling constant, and $f_{p, n}$ is the frequency of the clock at point $T_{p, n}$

$T_{p, n}^{\prime}=T_{p, n}+C f_{p, n}$

This assumes that the change in power measurement due to varying clock frequency simply results in an 'offset' of the measured power. This assumption is also validated in [18], where a 'sliding match' method is used to compensate for the effect of the varying clock on power consumption traces.

To further test this assumption, the mean and standard deviation of each power trace was plotted for the operating frequency $f_{n}$, where $f_{n}$ varies by the 'extended' range given in Table 1 . The results are shown in Fig. 6.

Over a somewhat limited range the assumption appears to hold: for example over the range of approximately $7.2 \mathrm{MHz}-8.1 \mathrm{MHz}$ the mean varies linearly with frequency, and the standard deviation is constant. Thus in this range there is no scaling of values, just a bias which must be corrected for. Over the extended frequency range it would appear some scaling of points is required, as the standard deviation is also varying with frequency.
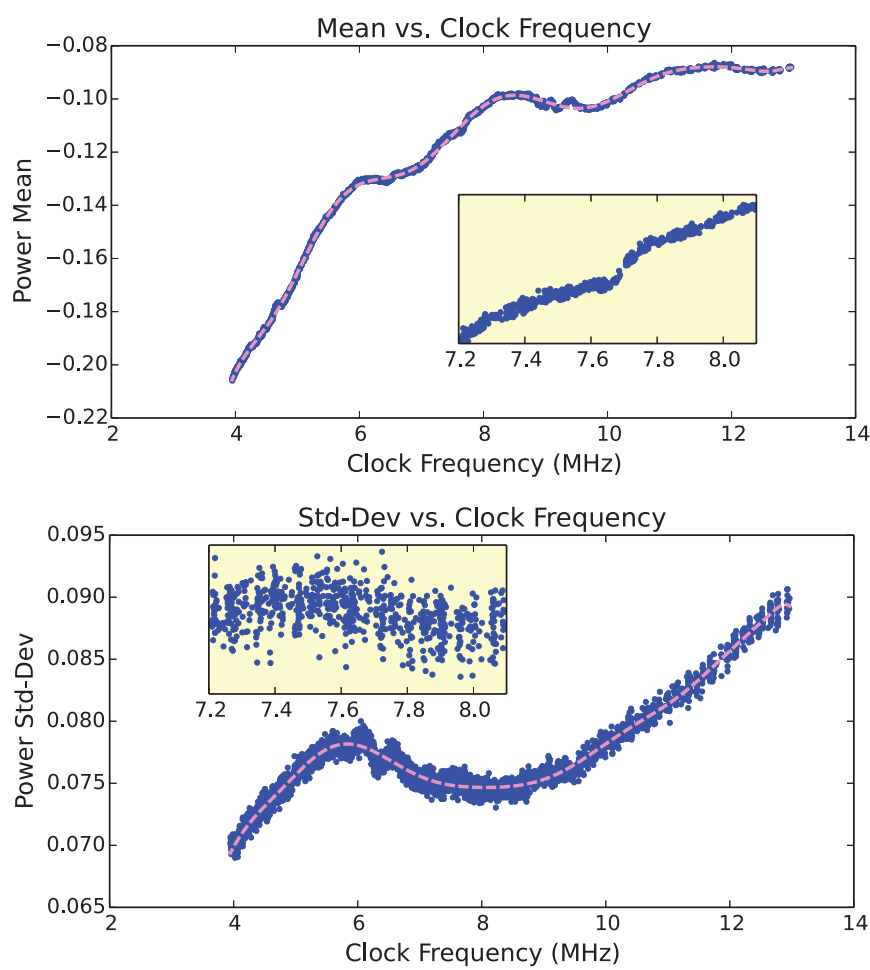

Fig. 6 Plot of the trace mean standard deviation compared to operating frequency of the microcontroller, from $3.9-$ $13 \mathrm{MHz}$. Inset details $7.2-8.1 \mathrm{MHz}$ range. Dashed line shows the $\hat{\mu}(f)$ and $\hat{\sigma}(f)$ functions used in (4) and (5).

Four additional preprocessing methods are proposed here; all five methods will be tested by comparing the results of the Correlation Power Analysis (CPA) attack over several frequency ranges.

First, two methods which do not require knowledge of the frequency of operation are proposed. The most basic simply scales all traces to be zero-mean, which again would be expected to only work over a limited frequency range:

$T_{p, n}^{\prime}=T_{p, n}-\hat{\mu}_{T_{n}}$

This can be improved by also scaling by standarddeviation, which should improve performance over a wider range. This will convert the distribution of each trace to be the 'standard normal' distribution. Applying this zero-mean, unit variance normalization (MVN) to side-channel attacks has already been used to improve the applicability of template-based attacks beyond the specific hardware which generated the template[11]. This preprocessing is given by:

$T_{p, n}^{\prime}=\frac{T_{p, n}-\hat{\mu}_{T_{n}}}{\hat{\sigma}_{T_{n}}}$

The main downsides of these methods is they require the frequency be constant over the entire length 
of the trace. Method (1) was proposed in [15] as it could function where the frequency varies per clock cycle. To accomplish this same goal, we will define an estimate function $\hat{\mu}(f)$ which provides an estimate of the mean of the power trace for a known frequency f, and similarly $\hat{\sigma}(f)$ which provides an estimate of standard deviation of the power trace. These functions are simply $15^{\text {th }}$ order polynomial curves fitted to the data in Fig. 6. The plots of both functions are shown in Fig. 6 as well.

Repeating (2) but with $\hat{\mu}$ being a function of $f$, and not simply calculated over the entire trace:

$T_{p, n}^{\prime}=T_{p, n}-\hat{\mu}\left(f_{p, n}\right)$

And similarly for (3):

$T_{p, n}^{\prime}=\frac{T_{p, n}-\hat{\mu}\left(f_{p, n}\right)}{\hat{\sigma}\left(f_{p, n}\right)}$

Fig. 7 shows traces before and after preprocessing, using (5) - note the alignment in the time domain of all the traces due to synchronous sampling, despite the varying clock of the DUT.

Even with synchronous sampling, some trace resynchronization may be required. In this case if the sampling was started and then the clock speed changed, the traces had slight misalignment. It is assumed this comes from either the microcontroller delaying execution during the frequency change, or errors in the sampling ADC as the clock frequency changes. The synchronous sampling still greatly simplified the further resynchronization required, as all traces were within 3 samples (clock cycles) of each other. If the sampling was started after the clock frequency speed changed, no resynchronization was required, despite the DUT running at different frequencies.

\subsection{Results}

The PGE of the CPA attack on an 'extended' frequency variation is shown in Fig. 8. Note from Table 2 the more widely varying 'extended' range of frequency variation has slightly worse performance than the 'drift' range, thus the varying clock does diminish performance. With the crystal oscillator, performance is similar to the 'extended' range. One would expect it to be similar to the 'drift' range instead, since the frequency is not varying. Thus is assumed to be caused by the external oscillator circuitry in the AVR microcontroller resulting in more noise on the trace measurement. Thus using an internal RC oscillator can actually result in lower-noise measurements compared to an external oscillator.

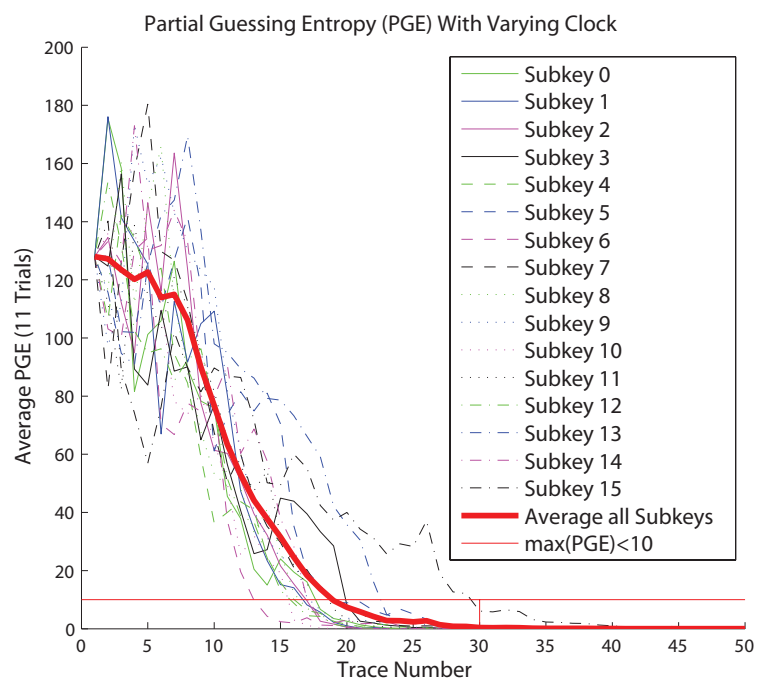

Fig. 8 Results of a CPA attack on a device with oscillator frequency randomly varying between $3.9 \mathrm{MHz}-13 \mathrm{MHz}$ on each encryption, and no trace synchronization being performed. The Byte $N$ refer to the subkey Partial Guessing Entropy(PGE), Average refers to the average of all 16 subkeys. $\max (P G E)<10$ shows the metric used in Table 2 .

Attempting to attack anything besides the 'crystal' range with measurements taken by a standard asynchronous oscilloscope fails. The PGE does not significantly improve over the range of trace measurements, even for the 'drift' range. The results of Fig. 21 demonstrate this in practice.

As previously mentioned a number of preprocessing methods are also tested, with final results shown in Table 2. The details of the PGE metric are provided in Section 2.2. The $\max (P G E)<10$ point is shown in figures as the horizontal line at $P G E=10$. It can be noted that over a narrow frequency range no preprocessing is required: the 'drift' range has no improvement using any preprocessing method. Only the 'extended' range shows significant improvement in attack performance by using preprocessing, and even then the method makes little difference.

These results suggest that details of the preprocessing are not too critical, and would also validate previous work such as [15] which indicate a simple frequencydependant bias is sufficient. In cases where the frequency is constant over the entire trace, it is sufficient to simply subtract the mean of each trace from itself, forcing the trace to be zero-mean.

Considering the extremely large range the oscillator was varied over $(3.9 \mathrm{MHz}-13 \mathrm{MHz})$, these results show that synchronous sampling is a simple method of attacking the varying clock (VC) countermeasure. 
Table 2 The number of traces for the Partial Guessing Entropy (PGE) of the CPA attack to be $<10$ is given in this table, where the traces have been preprocessed by different methods.

\begin{tabular}{lcccccc}
\hline Clock & $\mathbf{T}_{n}$ & $\mathbf{T}_{n}+C f_{n}$ & $\mathbf{T}_{n}-\mu_{T_{n}}$ & $\frac{\mathbf{T}_{n}-\mu_{T_{n}}}{\sigma_{T_{n}}}$ & $\mathbf{T}_{n}-\hat{\mu}\left(f_{n}\right)$ & $\frac{\mathbf{T}_{n}-\hat{\mu}\left(f_{n}\right)}{\hat{\sigma}\left(f_{n}\right)}$ \\
\hline Extended & 93 & 32 & 28 & 30 & 28 & 30 \\
Narrow & 23 & 19 & 16 & 15 & 15 & 15 \\
Drift & 12 & 12 & 12 & 13 & 12 & 12 \\
Crystal & 29 & 29 & 29 & 30 & 30 & 29 \\
\hline
\end{tabular}

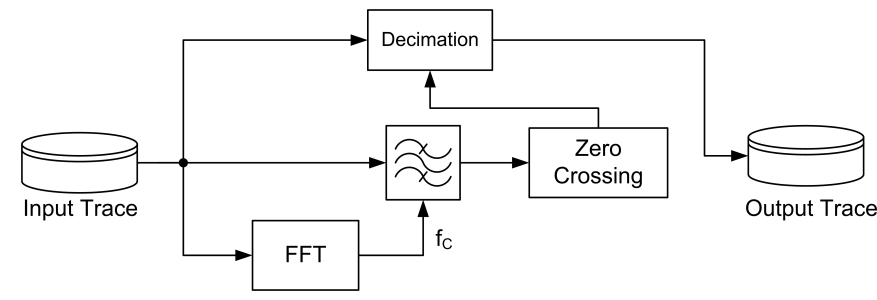

Fig. 9 Previous work on trace compression can be considered a simple example of clock recovery. Here the trace compression is performed by simply detecting zero-crossing events which correspond to clock edges, and only storing those points.

\section{Clock Recovery as Preprocessing}

If we consider the case of asynchronous sampling, where the sample rate is infinitely fast, the synchronous sampling method would be equivalent to performing trace compression which is keeping a single point per clock sample[8]. Practically of course this means simply sampling 'fast enough' for a specific target; looking at Fig. 1, we can see for the AtMega48A sampling at $312 \mathrm{MS} / \mathrm{s}$ should be sufficient.

In this work, recovering the clock is done by filtering the recovered signal around the fundamental frequency component. This method is used since it is possible to implement in both software and hardware. In particular the hardware implementation will be used for real-time recovery of a device clock for synchronous sampling and glitch generation.
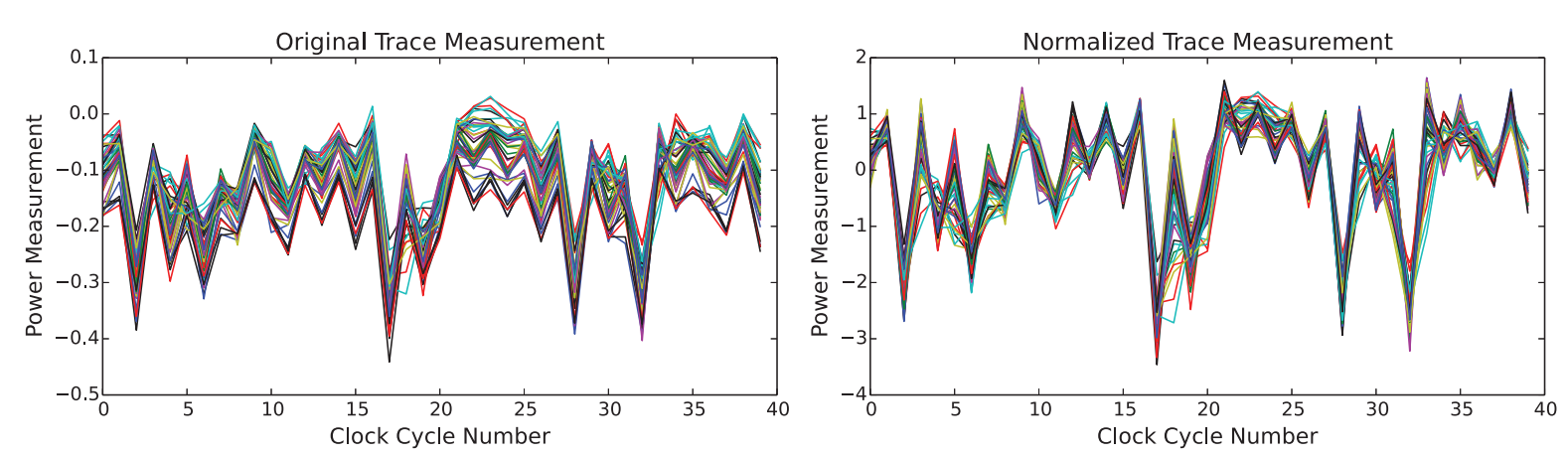

Fig. 7 Traces can be normalized by (5) before passing to a standard CPA attack to remove the effect of varying operating frequency.
Fig. 9 shows a block diagram of the clock recovery and decimation logic. A FFT is used on the input trace to determine the operating frequency of the device, where it is assumed the operating frequency results in the largest harmonic component. Systems with multiple oscillators may require a more complex selection logic.

A $5^{\text {th }}$ order IIR Butterworth bandpass filter with a center frequency $f_{C}$ processes the received data, where $f_{C}$ is selected as the device operating frequency. The passband of the filter is configured to have a bandwidth of $20 \%$, where the bandwidth for a filter with a passband from $f_{L}$ to $f_{H}$ is given by $(6)$.

$B W=2 \frac{f_{H}-f_{L}}{f_{H}+f_{L}}$

The sample corresponding to a clock edge is selected based on a zero-crossing detection of the filtered output. This means the effective sample rate becomes $2 \times$ the device clock frequency, since two zero-crossings are produced for every cycle.

The results of a CPA attack against a system where the clock is constant, i.e. the crystal range, is shown in Fig. 10. This comparison shows that the clock recovery logic can reduced the trace size with minimal impact on attack performance. Using integer decimation by comparison results in a performance penalty on the attack results. 


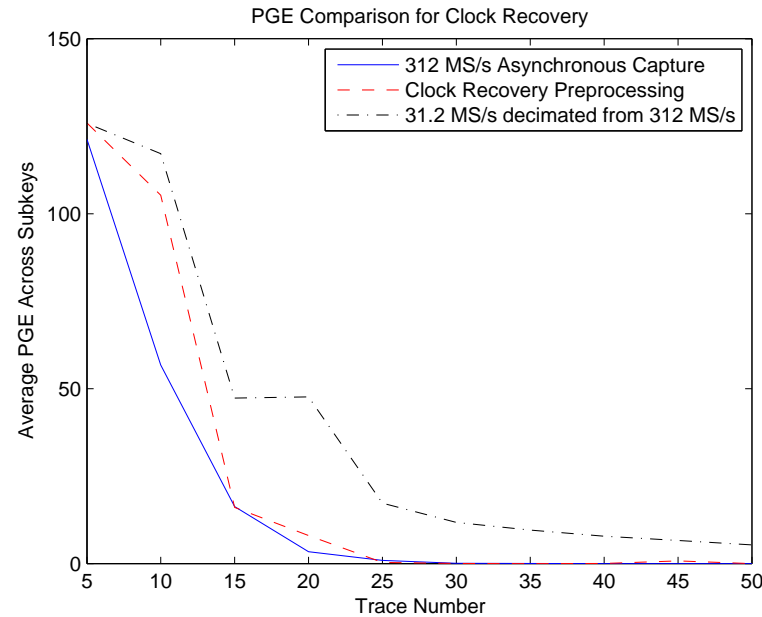

Fig. 10 All results come from same traces, captured on $\mathrm{Pi}-$ coScope $6403 \mathrm{D}$ at $312 \mathrm{MS} / \mathrm{s}$. Each trace in the raw file has 31888 points, the clock recovery version has 1500 points, and the decimated trace file has 3188 points.

\subsection{Clock Recovery with Varying Clock}

Initially, the internal RC oscillator is used without any explicit random frequency generation. The RC oscillator does randomly drift about $\pm 0.5 \%$ during operation as measured in Table 1. Measurements taken with a standard oscilloscope fail to recover the key as shown in Fig. 21, even after 1000 trace measurements. When the clock is stable the standard oscilloscope recovers the key in $<20$ traces, as in Fig. 1. Thus the small amount of clock variation causes the CPA attack to fail, despite the starting point having perfect synchronization. If instead we use clock recovery algorithm from Fig. 9, the results are as in Fig. 11. The CPA attack is successful and with similar success to the original setup!

Next, the 'narrow' frequency range in Table 1 is used for clock recovery, which has a center frequency of $7.66 \mathrm{MHz}$. The frequency was varied approximately $\pm 5.5 \%$. Fig. 12 gives the results of the CPA attack on this system.

Finally, the 'extended' clock frequency range which varies from $3.9 \mathrm{MHz}-13 \mathrm{MHz}$ is used, which has a center frequency of $7.21 \mathrm{MHz}$. Fig. 13 gives the results of the CPA on this setup.

These results show that the CPA attack remains successful on all targets, despite the highest operating frequency being over $3 \times$ the lowest operating frequency.

\section{Clock Recovery Hardware}

In many devices the clock is not available externally, meaning additional work is required to perform synchronous sampling. In side-channel analysis, it was pre-

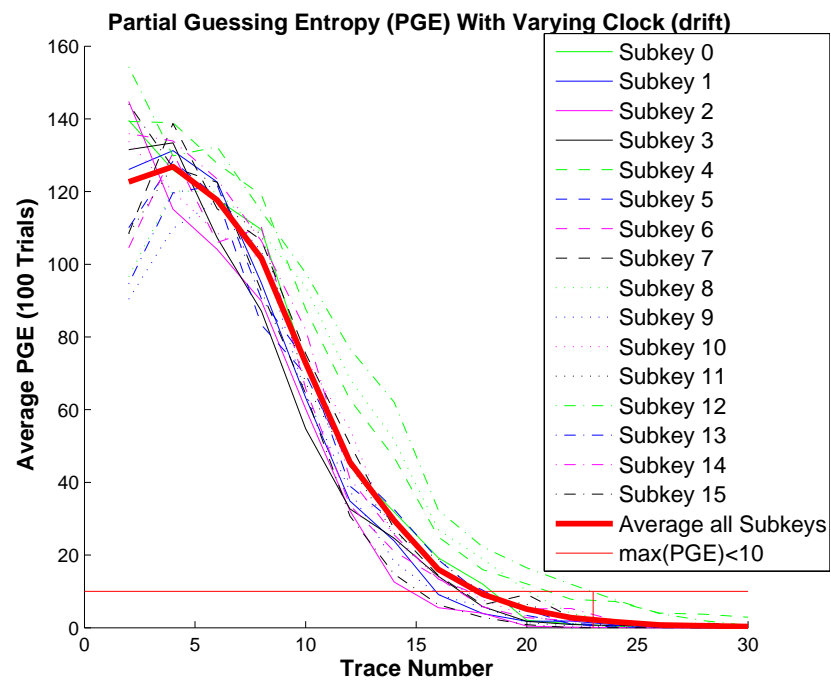

Fig. 11 Results of a CPA attack on a device with an internal $\mathrm{RC}$ oscillator, where the oscillator frequency changes $\pm 0.5 \%$ during operation due to drift, and the clock is not externally available, but clock recovery as a preprocessing is used. Average refers to the average of all 16 subkeys. Subkey plot legend same as in Fig. 8.

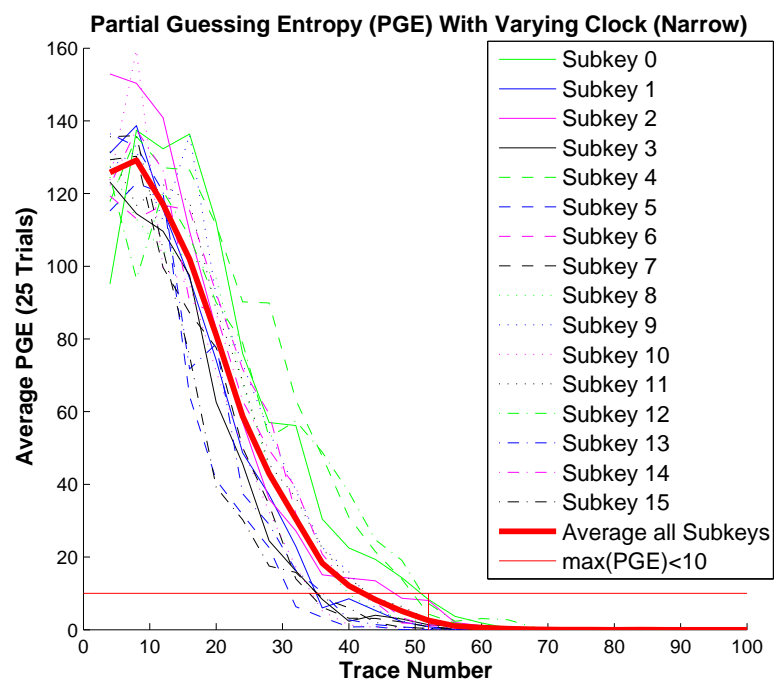

Fig. 12 Results of a CPA attack on a device with an internal $\mathrm{RC}$ oscillator, where the oscillator frequency changes $\pm 5.5 \%$ during operation, and the clock is not externally available, but clock recovery as a preprocessing is used. Average refers to the average of all 16 subkeys. Subkey plot legend same as in Fig. 8.

viously demonstrated how to force an internal oscillator to lock to an external signal [16]. This was used to stabilize the internal RC oscillator and improve trace synchronization, but the same method could be used to generate the reference clock for synchronous sampling. This will fail if the device itself is varying the clock frequency, so instead clock recovery must be used 


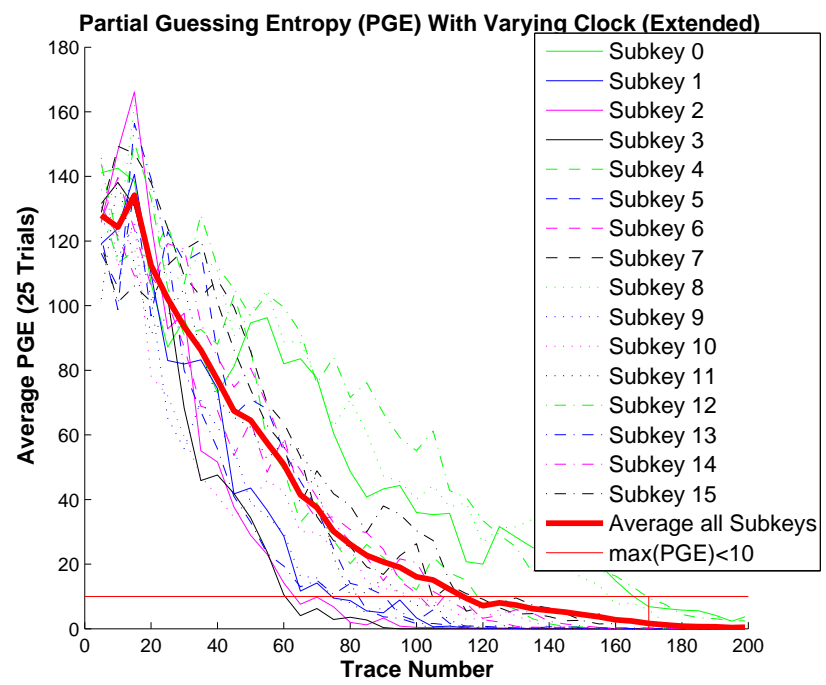

Fig. 13 esults of a CPA attack on a device with an internal $\mathrm{RC}$ oscillator, where the oscillator frequency changes $-45 \%$ to $+80 \%$ during operation, and the clock is not externally available, but clock recovery as a preprocessing is used. Average refers to the average of all 16 subkeys. Subkey plot legend same as in Fig. 8.

to generate a copy of the clock. The idea of clock recovery is not new - in communications electronics this has been used for many years to synchronize a receiver clock to a transmitter clock over long distances[4].

The basic method used here for clock recovery is to filter the power signal so that only the fundamental frequency from the internal oscillator is left. This can then be amplified and turned into a digital signal. To prevent glitches from resulting at the output a PLL is used to provide a clean digital signal. Details of this hardware design and results of side-channel analysis tests will be presented next.

\subsection{Hardware Design}

A block diagram of the system is given in Fig. 14, for a complete schematic see Appendix A. A Low Noise Amplifier (LNA) is placed on each side of the bandpass filter $(\mathrm{BPF})$, the BPF selecting the fundamental frequency from the power signal. The output of the final LNA is limited to logic levels and fed into the Phase Lock Loop (PLL) block. The PLL used is a single-chip solution, the Texas Instruments CDCE906 device which integrates the Voltage Controller Oscillator (VCO), Phase Detect (PD), loop filters, and frequency dividers into a single package. For an introduction to PLLs the reader is referred to [2].

Fig. 15 shows an example of recovering an internal oscillator on an Atmel AVR ATMega48A device. With

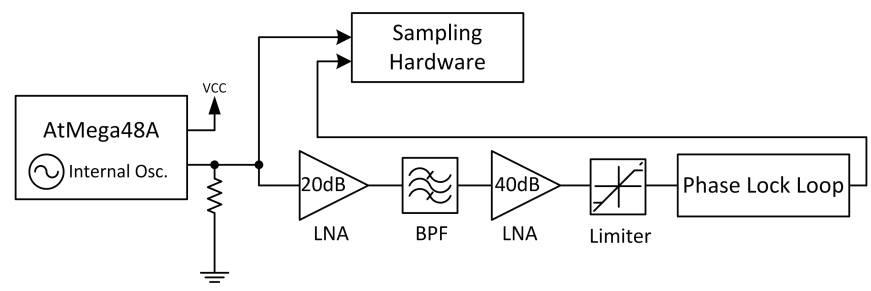

Fig. 14 Clock Recovery Block Diagram.

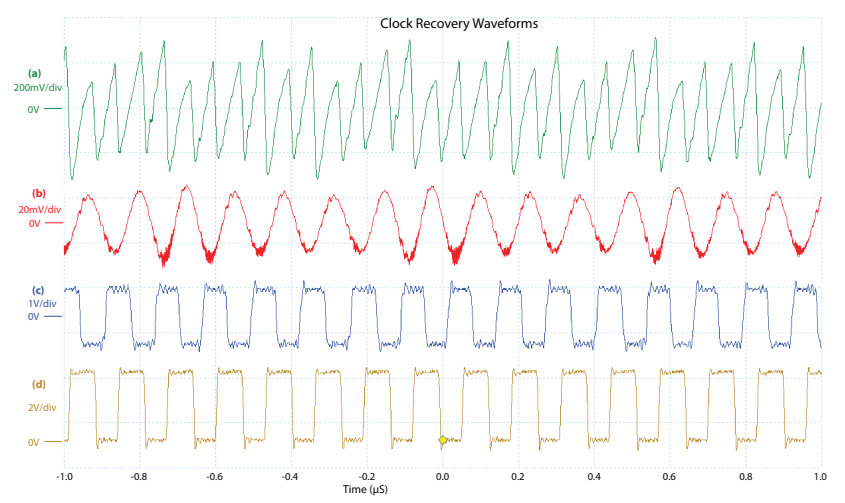

Fig. 15 Recovery of $7.7 \mathrm{MHz}$ Internal RC Oscillator on ATMega48A. (A) is the amplified power trace after the LNA. (B) is the output of the band-pass filter, and (C) is the output of the limiter, which generates a logic-level signal. The output of (C) can be passed through a PLL to further stabilize the signal. In (D) the actual RC oscillator output is shown, note the perfect alignment of the recovered signal (C) and internal $\mathrm{RC}$ oscillator (D).

this device it is possible to switch on a 'clock out' pin, which allows measurement of the internal RC oscillator signal. The clock recovery logic works equally well with this pin enabled or not, but enabling the pin allows comparison of the recovered clock to the internal oscillator.

\subsection{Filter Design}

The design of the band-pass filter (BPF) is critical for the success of the clock recovery, details of the design process are given in Appendix A. Selection of the passband is based on the frequency of the internal oscillator for the device under attack. If this frequency is not known it can typically be found by viewing the frequency spectrum of the device during operation.

Careful consideration must be given for the group delay of the filter, which changes over frequency. As an example the $6.5 \mathrm{MHz}-8.5 \mathrm{MHz}$ BPF used for the ATMega48A device is shown in Fig. 16. The group delay, which is usually measured in time units or phase degree, has been scaled by the frequency to give us a group delay in 'clock cycles'. The group delay will cause synchronization errors between traces if the frequency 


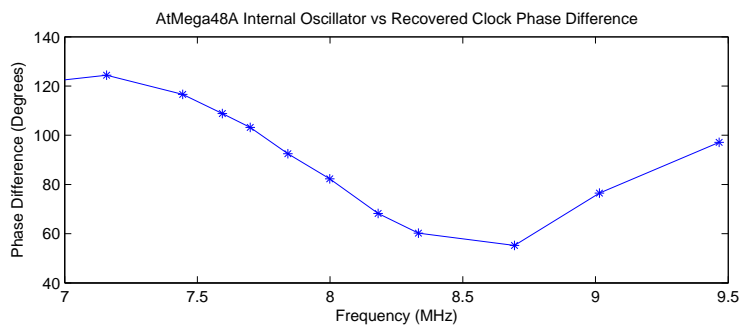

Fig. 17 As the phase difference changes, the alignment of measurements is compromised, requiring more traces. This figure shows the measured phase difference for the overall system, i.e. phase difference between the $\mathrm{RC}$ oscillator on the AVR and the final recovered clock. A Bessel analog filter (as given in Appendix A) is used here, results are from measurement.

of the DUT oscillator changes, since the delay through the filter varies with frequency.

For more detail, the delay between the actual internal $\mathrm{RC}$ oscillator and the recovered clock is plotted in Fig. 17 over a more limited range. Here the delay is measured in degrees, where $360^{\circ}$ equals one clock cycle. This figure comes from measurements of the final implemented system, whereas Fig. 16 is based on simulations of just the BPF.

Three methods to reduce this error can be used. First, the type of analog BPF should be matched with the DUT. If the frequency of the oscillator varies only a tiny amount, it would be possible to use a Chebyshev filter with the better attenuation performance. If the DUT oscillator frequency will vary a filter with better group delay performance could be used such as the Bessel. The second way to reduce this error is to measure the frequency during each trace acquisition, and shift the recorded waveform by the known group delay of the filter at this frequency. Finally a standard trace synchronization algorithm can be used to synchronize all such traces.

\subsection{Results of CPA Attack}

The AtMega48A platform is used again for this evaluation. The 'external clock' output is disabled during these tests - the AVR driving the IO pin at the clock frequency results in a very strong fundamental harmonic on the power trace, which results in a better signal for the PLL to lock onto. Such a system would be unrealistic since real systems would not be driving an arbitrary IO pin causing this strong fundamental.

The complete setup with clock recovery module, OpenADC capture hardware, and target is shown in Fig. 18.

The test setup is almost identical to that of Section 4.1 , where clock recovery is done via processing of traces capture asynchronously. Again initially only a small frequency variation due to drift of about $\pm 0.5 \%$ during operation is used, as measured in Table 1. With synchronous sampling with clock recovery as proposed in this paper, the results are as in Fig. 19. The CPA attack is successful without any special processing of the traces.

Next, the 'narrow' frequency range in Table 1 is used for clock recovery, which has a center frequency of $7.66 \mathrm{MHz}$. Fig. 20 gives the results of the CPA attack on this system. The reduced performance is mainly due to the phase delay of the clock varying with frequency, as in Fig. 17. When the clock is directly available and not obtained through clock recovery, as in the results of Table 2, the 'narrow' frequency range has similar performance to the 'drift' range.

The 'extended' clock frequency range of $3.9 \mathrm{MHz}-$ $13 \mathrm{MHz}$ could not be recovered using the simple filtering method. This is due to the fact that the $3^{\text {rd }}$ harmonic of $3.9 \mathrm{MHz}$ will be at $11.7 \mathrm{MHz}$, which would fall within the bandpass filter bandwidth. Using clock recovery on a very widely varying clock would require a tunable filter which follows the fundamental frequency.

Note that comparing the results to the softwarebased clock recovery from Section 4.1 shows that asynchronous sampling has better performance, it is assumed due to the ability to generate an ideal filter, instead of being limited by physical component selection. The clock recovery method is still useful when it is desired to use synchronous sampling due to the reduced sample rate requirement compared to capturing asynchronously and later processing the data. For fault injection processing the data after capture is not useful, since real-time information is required. The next section will concentrate on the use of clock recovery for these cases.

\section{Fault Injection}

For injecting faults into an embedded system, having a clock which is phase-locked to the device clock allows more precise temporal location selection. If triggering must count a certain number of clock cycles for example, this is difficult to do over long periods due to drift in either the device clock or the instrument clock. If the device clock itself is used, it is trivial to count over a large number of cycles with great accuracy.

Previous work has looked at either disabling the switch to an unstable clock [19], or forcing the internal clock to lock to an external clock[16]. These methods are highly dependant on a specific system design; a device may instead always come up on an internal 
This is the authors version of an article accepted into the Journal of Cryptographic Engineering.

The final publication is available at www.springerlink.com. 10.1007/s13389-014-0087-5
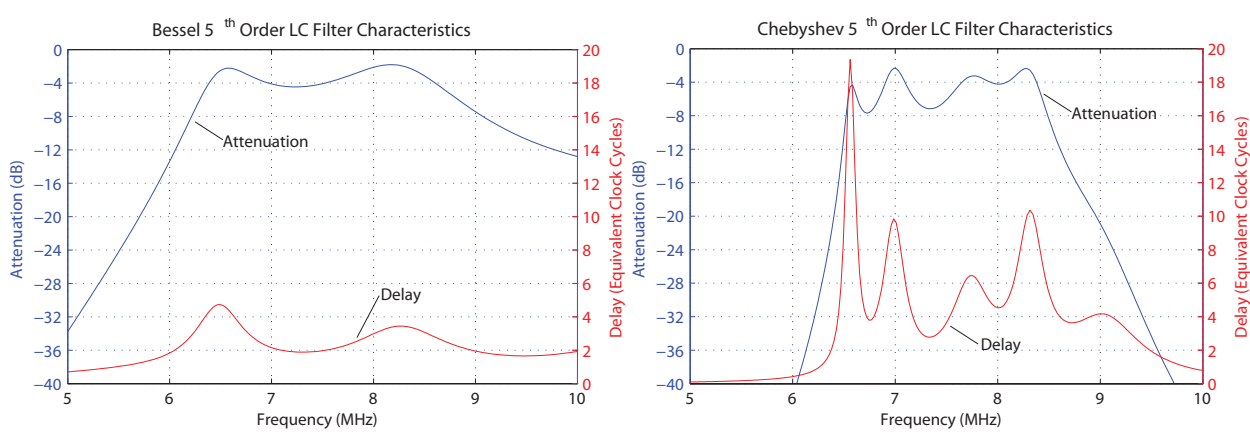

Fig. 16 Choice of filter type means a choice between better group delay performance and better attenuation outside the passband. Two examples are given here: a Chebyshev filter and a Bessel filter, both 5th order made from discrete LC components. Results are from simulation.

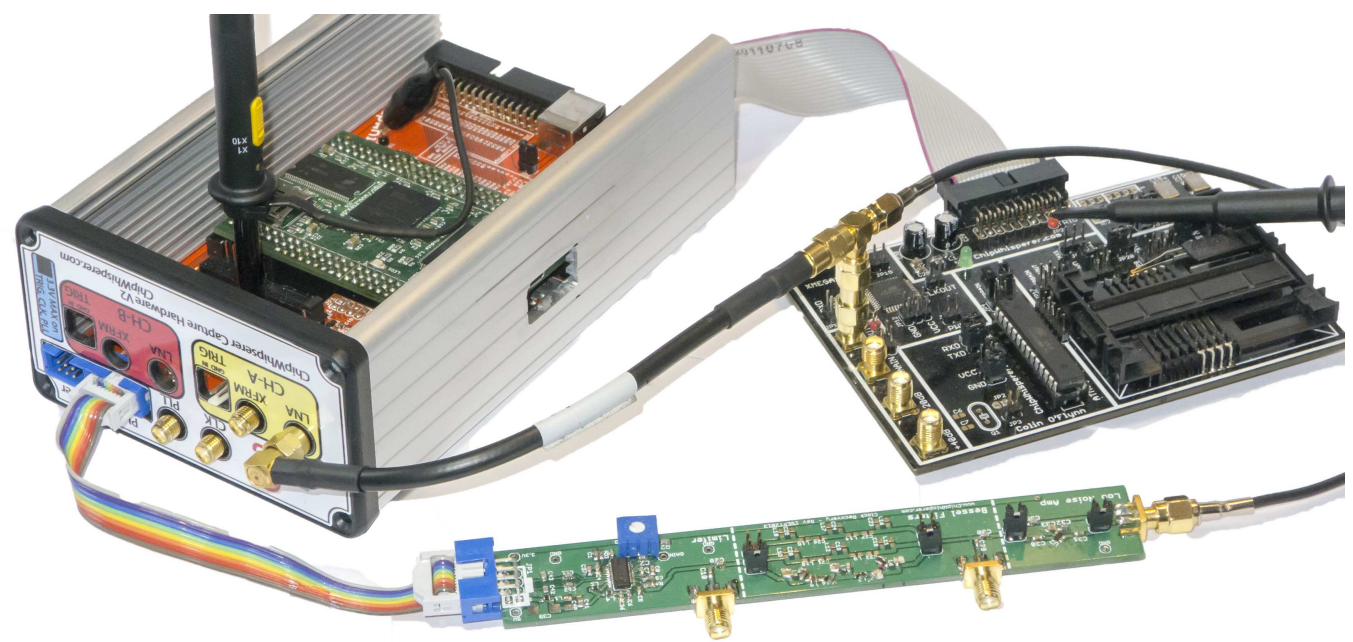

Fig. 18 Test Setup for side-channel analysis with clock recovery of internal oscillator on ATMega48A. The oscilloscope is used to measure recovered clock frequency. The long board center-front performs amplification, filtering, and limiting. The PLL is located inside the capture hardware on the left-hand side. The back right board is the AtMega48A target.

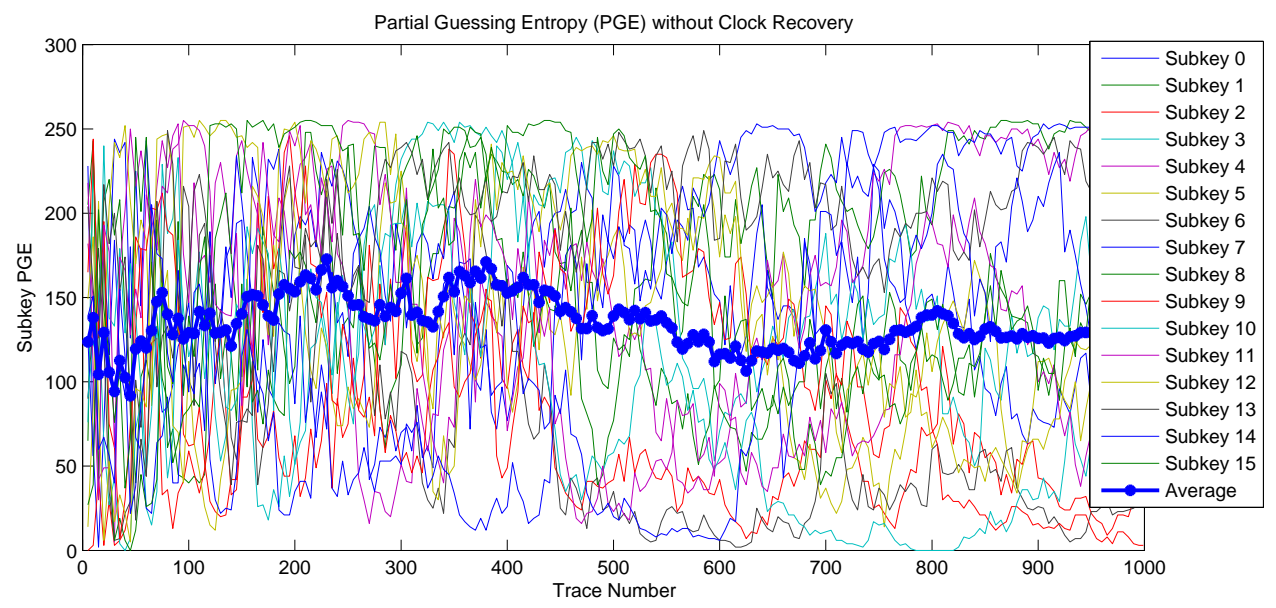

Fig. 21 Results of a CPA attack on a device with an internal RC oscillator, where the oscillator frequency changes $\pm 0.5 \%$ during operation due to drift, and a standard asyncronous oscilloscope samples the device at $312 \mathrm{MS} / \mathrm{s}$. Average refers to the average of all 16 subkeys. 


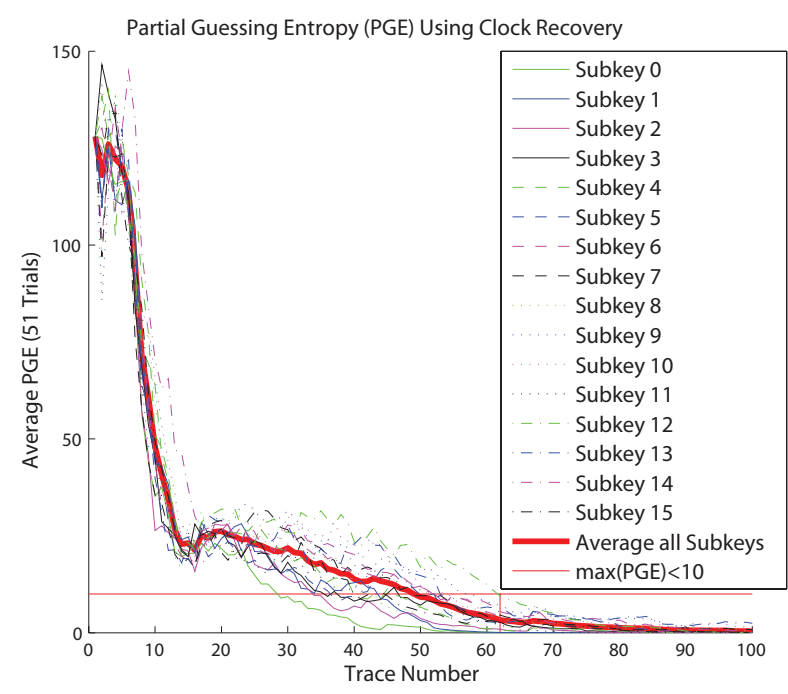

Fig. 19 Results of a CPA attack on a device with an internal $\mathrm{RC}$ oscillator, where the oscillator frequency changes $\pm 0.5 \%$ during operation due to drift, and the clock is not externally available, but clock recovery with synchronous sampling used. Average refers to the average of all 16 subkeys. Subkey plot legend same as in Fig. 8.

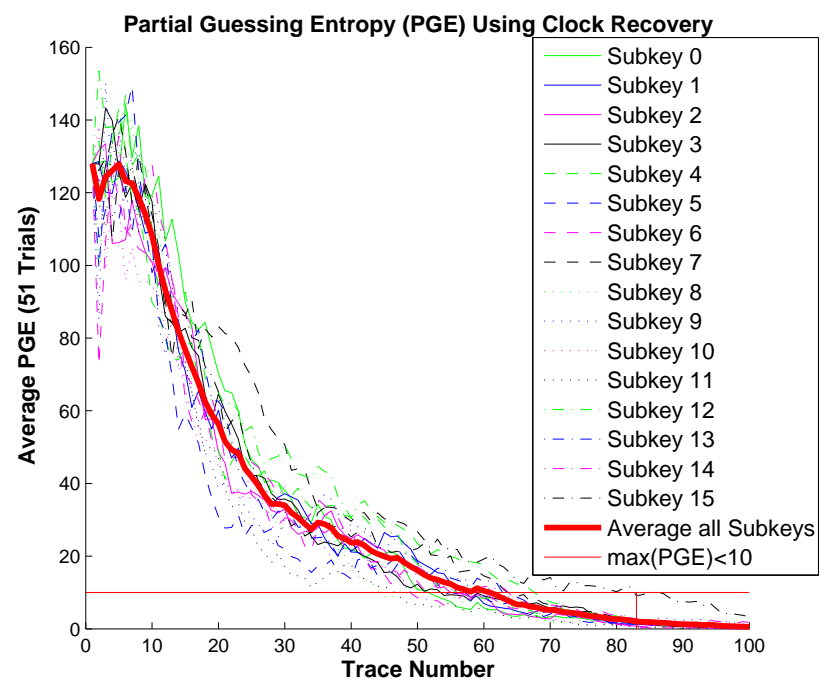

Fig. 20 Results of a CPA attack on a device with an internal $\mathrm{RC}$ oscillator, where the oscillator frequency changes $\pm 5.5 \%$ during operation, and the clock is not externally available, but clock recovery with synchronous sampling used. Plot legend same as in Fig. 8.

oscillator, making it impossible to keep it running on the external clock.

Having a phase-locked clock means glitches can even be inserted at specific portions of the device clock cycle. These glitches could be power, EM[14], or laser/optical[17]. We are assuming there is no external clock in this work, thus are ignoring clock perturbations as a valid glitch. This work will use power glitches as a demonstration of the usefulness of maintaining a phaselocked reference, such as is derived by the clock recovery scheme.

In addition a triggering mechanism that depends on waveforms in the analog data is demonstrated. How changes in operating frequency affect the triggering reliability is also explored, and it will be demonstrated that synchronous sampling provides a highly reliable data source for this trigger.

\subsection{Sum of Absolute Difference Trigger}

To inject a fault at a specific location, a pattern detection trigger called the Sum of Absolute Difference (SAD) is used. The implementation of the SAD comes from the ChipWhisperer system [13]. In this implementation 128 input samples, $\mathbf{T}$, are continuously compared to a 128 point reference waveform, $\mathbf{R}$, using (7). If the input was exactly the same as the reference waveform, the output of (7) would be 0 . Normally the trigger condition is simply when the output of (7) falls below some point.

$S A D=\sum_{p=0}^{127}\left|T_{p}-R_{p}\right|$

If the data $\mathbf{T}$ has already been recorded (e.g. for resynchronizing recorded data), the form of (8) can be used. In this form an 'offset' parameter $m$ is added, which slides the comparison window across all points in the recorded trace.

$S A D(m)=\sum_{p=0}^{127}\left|T_{p+m}-R_{p}\right|$

To determine the effect of varying clock frequency, a SAD reference waveform $\mathbf{R}$ will be compared to a recorded power trace $\mathbf{T}$, where the same operation is occurring in both $\mathbf{T}$ and $\mathbf{R}$. The frequency that the target is operating at when $\mathbf{T}$ is recorded varies, and the output of the SAD equation (8) is calculated. It is known a priori that when $m=0$ the operations in both waveforms should be synchronized. Thus we would expect the following:

$\underset{m}{\arg \min }(S A D(m))=0$

To determine the margin for the SAD trigger level, the minimum value of (8) is found when the offset is not zero, i.e. for all the wrong alignments of $\mathbf{R}$. This is plotted against frequency in Fig. 22 - the distance 


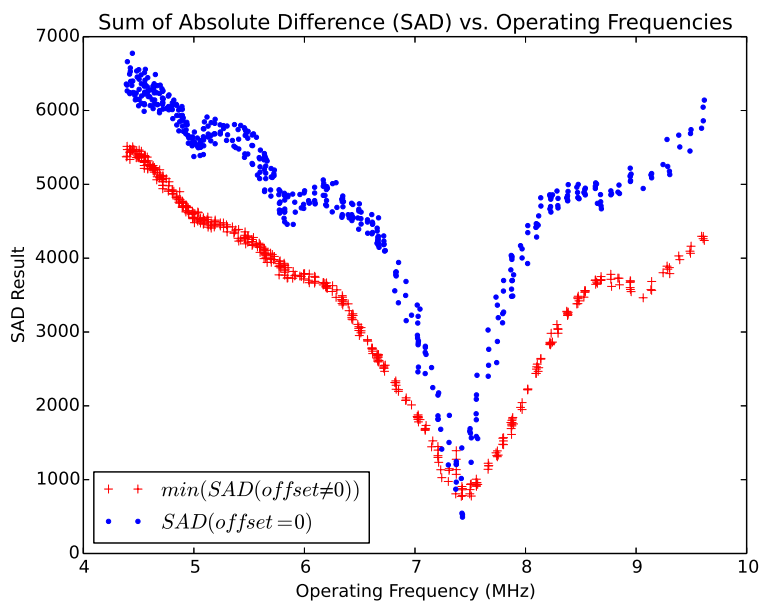

Fig. 22 Output of (8) for the offset $m$ being zero or nonzero. When the offset $m=0$, this means the SAD output for the correct alignment of traces. When $m \neq 0$, this means the best possible SAD output for incorrect alignment of traces. Data sampled asynchronously at $312.5 \mathrm{MS} / \mathrm{s}$.

between the two groups indicates the margin available. This uses a normal asynchronous capture, and note the SAD trigger would only function at a very narrow window around the reference trace waveform, which was captured when running at about $7.4 \mathrm{MHz}$.

By comparison, if we use synchronous sampling the $\mathrm{SAD}$ triggering is able to reliably detect the triggering point for $\mathbf{T}$ being recorded with a device frequency between $4.2 \mathrm{MHz}-13 \mathrm{MHz}$, even though the reference $\mathbf{R}$ was recorded at a different device operating frequency (about 7.6 MHz). At the extreme lower end of the operating frequency range the $\mathrm{SAD}$ triggering is not reliable, as around $3.9 \mathrm{MHz}$ it would select the wrong triggering point.

For using the SAD triggering, hardware clock recovery is required if the device frequency is not constant. We will next consider not only the triggering of glitches, but the actual parameters defining the glitches as the device frequency varies.

\subsection{Fault Injection and Target Code}

For generation of faults, power glitching is used. A MOSFET is used across the power pins of the chip; the MOSFET forms a voltage divider with the shunt resistor being used for side channel power analysis measurement, and allows quickly dropping the voltage on the VCC pin. An example of the glitch waveform is shown in Fig. 24.

This setup allows power consumption to be monitored (required for the SAD trigger) along with monitoring the glitch status. The width and offset of the

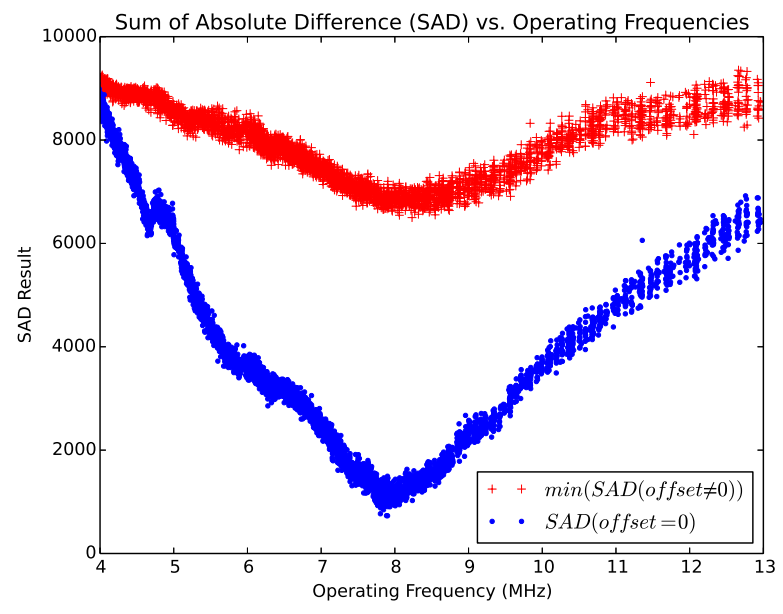

Fig. 23 Output of (8) for the offset $m$ being zero or nonzero. When the offset $m=0$, this means the SAD output for the correct alignment of traces. When $m \neq 0$, this means the best possible SAD output for incorrect alignment of traces. Data sampled synchronously at $4 \times$ device clock.

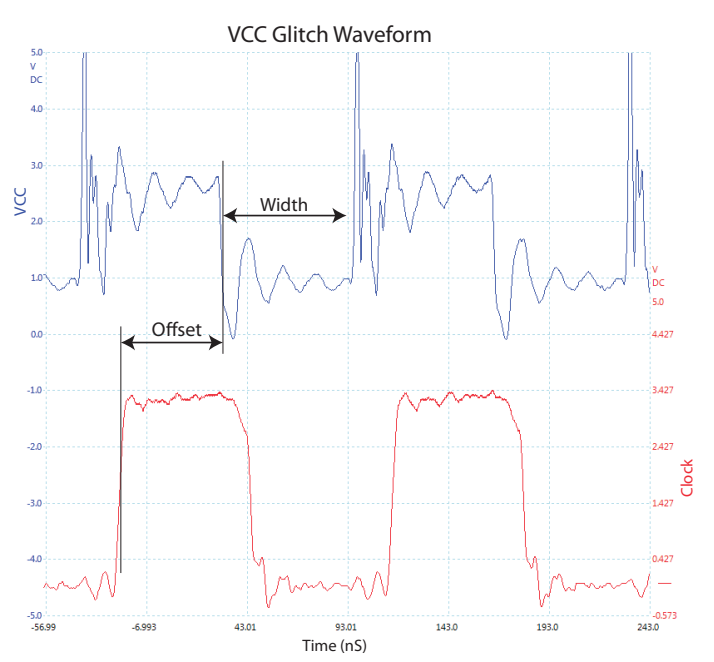

Fig. 24 The VCC glitch inserted into the AtMega48A device for this test is derived from a source clock. The glitch width and offset are a function of that source clock, see [13] for details.

glitch is controlled via the ChipWhisperer system. The glitch width and offset is based on a percentage difference from the 'source clock'. If the device clock is known, this allows the width and offset to scale with changes in frequency, and ensures perfect synchronization of glitch location relative to clock edges. The ChipWhisperer system has high resolution on the glitch width and offset, having approximately $100 \mathrm{pS}$ resolution on these options.

Where the source clock isn't known, i.e. without using clock recovery, an asynchronous clock is instead used to generate the glitch width and offset. In this case the glitch offset will occur relative to the trigger event, 
however the glitch parameters do not scale with device frequency, since the device frequency is not known.

Listing 1 The source C code for the AtMega48A on which the glitch is tested.

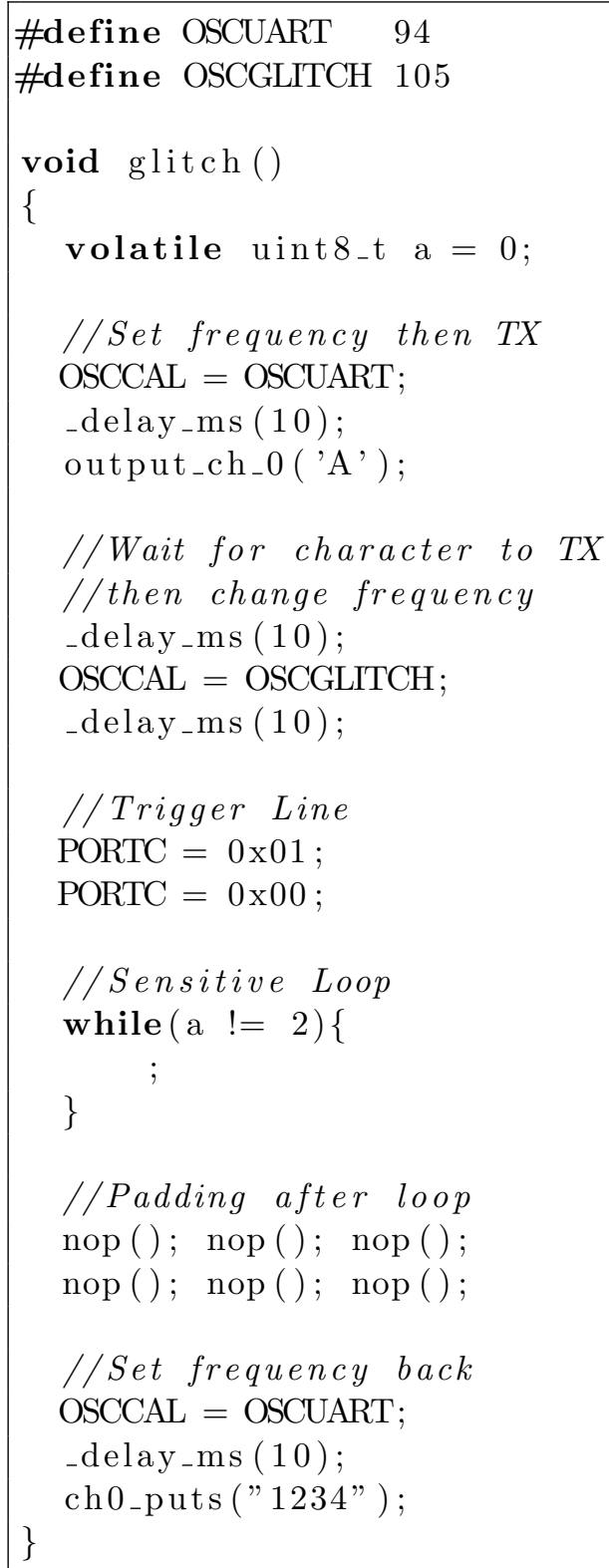

\subsection{Dependency on Target Frequency}

As previously mentioned, the use of clock recovery is required for the SAD triggering to function. To allow comparison of glitch insertion with and without clock recovery, the AtMega48A is also programmed to set an IO line high at the moment where a glitch should be inserted. The glitch can thus be triggered even if the SAD trigger cannot be used, although in real systems it's unlikely such a trigger would exist. This trigger oc-

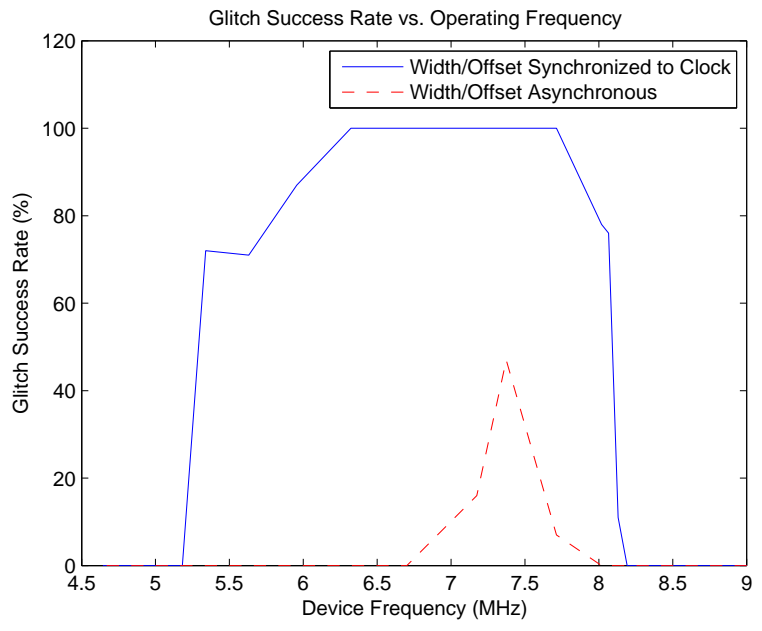

Fig. 25 Success of voltage glitching where the glitch parameters are fixed to maximize success at a clock frequency of $7.37 \mathrm{MHz}$, and then device is then operated at different frequencies.

curring at the moment of glitch insertion also means there is no error due to a differing number of device cycles between the trigger event and actual glitch, as would be the case if glitch insertion had a time-based offset from the trigger.

The code being glitched is shown in Listing 1, where a successful glitch is one which breaks out of the loop, without skipping past the padding. This allows a simple test to check if the glitch is causing the desired effect. A metric of the percent of glitches causing the desired effect that ' 1234 ' is printed is used to compare efficiency, which is averaged over 100 glitches.

The glitch offset and width is varied until what appears to be the maximum success rate is found. In one case the glitch width and offset scales with frequency (i.e. the device clock is fed into glitch generation), in the other the glitch width and offset is constant. The hardware is the same on both cases, again the AtMega48A device with an internal RC oscillator being used as the device clock.

It can be seen from the results of Fig. 25 that using the clock-synchronous glitch not only provides a more reliable glitch, but requires less tuning of parameters for operation over different frequencies in this example.

Considering that the synchronous capture provides the additional advantage of a useful SAD triggering system and the ability to easily count clock cycles from a trigger event, the clock recovery and synchronous capture method proposed here should have significant performance gains for fault injection. 


\section{Conclusions}

Synchronous sampling has already been demonstrated to be a useful tool in reducing the data complexity when working with side-channel analysis measurements $[10$, $12,13]$. It is know that compression of the power traces can be performed post-capture to reduce them to points of interest. Using synchronous sampling, however, eliminates the processing requirement, and makes triggering such as the Sum of Absolute Difference (SAD) mechanism reliable across operating frequency.

Synchronous sampling depends on the availability of the device clock, where many real devices contain an internal oscillator with no external signal. This paper has demonstrated how a 'clock recovery' technique can generate an external reference clock which is phase-locked to the internal oscillator of the device.

If the device under attack is varying the internal oscillator, this external clock will remain phase-locked to the true frequency. As synchronous sampling is measuring clock edges and not absolute time, this varying clock has very little effect of the success rate of an attack performed on these traces. The traces remain well synchronized despite the changing clock frequency, with the exception of a phase offset due to delay in the filter.

This recovered clock is also useful for fault injection, where it is desired to insert a fault at some specific clock cycle or portion of a clock cycle.

In addition to hardware-based solutions, this paper has also demonstrated the use of clock recovery with a standard asynchronous oscilloscope. This algorithm is of low complexity, and an implementation is available in the open-source ChipWhisperer project.

Acknowledgements Special thanks to funding provided by NSERC Canada Graduate Scholarship and OZ Optics. The authors appreciate the many constructive comments from anonymous reviewers which helped improve the final version of this paper.

\section{References}

1. Atmel Corporation: ATmega48A Datasheet

2. Banerjee, D.: PLL Performance Simulation and Design Handbook, 4th edn. Texas Instruments (2006)

3. Brier, E., Clavier, C., Olivier, F.: Correlation power analysis with a leakage model. Cryptographic Hardware and Embedded Systems - CHES 2004 pp. 135-152 (2004)

4. Costas, J.: Synchronous Communications. Communications Systems, IRE Transactions on 5(1), 99 -105 (1957). DOI 10.1109/TCOM.1957.1097490

5. Guilley, S., Khalfallah, K., Lomne, V., Danger, J.L.: Formal Framework for the Evaluation of Waveform Resynchronization Algorithms. In: Proceedings of the 5th IFIP WG 11.2 International Conference on Information Security Theory and Practice, WISTP'11, pp. 100-115.
Springer-Verlag, Berlin, Heidelberg (2011). URL http: //dl.acm.org/citation. cfm?id=2017824. 2017835

6. Kafi, M., Guilley, S., Marcello, S., Naccache, D.: Deconvolving Protected Signals. In: Availability, Reliability and Security, 2009. ARES '09. International Conference on, pp. 687 -694 (2009). DOI 10.1109/ARES.2009.197

7. Kocher, P., Jaffe, J., Jun, B.: Differential power analysis. In: Advances in Cryptology - CRYPTO' 99, pp. 388-397. Springer-Verlag (1999)

8. Mangard, S., Oswald, E., Popp, T.: Power Analysis Attacks: Revealing the Secrets of Smart Cards. Advances in information security. Springer (2008)

9. Massey, J.: Guessing and entropy. In: Information Theory, 1994. Proceedings., 1994 IEEE International Symposium on, pp. 204-(1994). DOI 10.1109/ISIT.1994.394764

10. Messerges, T.: Power Analysis Attacks and Countermeasures for Cryptographic Algorithms. Ph.D. thesis, University of Illinois at Chicago (2000)

11. Montminy, D., Baldwin, R., Temple, M., Laspe, E.: Improving cross-device attacks using zero-mean unitvariance normalization. Journal of Cryptographic Engineering 3(2), 99-110 (2013). DOI 10.1007/ s13389-012-0038-y

12. O'Flynn, C., Chen, Z.D.: A case study of Side-Channel Analysis using Decoupling Capacitor Power Measurement with the OpenADC. Lecture Notes in Computer Science 7743, 328-344 (2013)

13. O'Flynn, C., Chen, Z.D.: ChipWhisperer: An OpenSource Platform for Hardware Embedded Security Research. In: Constructive Side-Channel Analysis and Secure Design - COSADE 2014 (2014)

14. Quisquater, J.J., Samyde, D.: Eddy current for Magnetic Analysis with Active Sensor. In: Esmart 2002, Nice, France (2002)

15. Réal, D., Canovas, C., Clédière, J., Drissi, M., Valette, F.: Defeating Classical Hardware Countermeasures: A New Processing for Side Channel Analysis. In: Proceedings of the Conference on Design, Automation and Test in Europe, DATE '08, pp. 1274-1279. ACM, New York, NY, USA (2008). DOI 10.1145/1403375.1403684

16. Skorobogatov, S.: Synchronization method for SCA and fault attacks. Journal of Cryptographic Engineering 1(1), 71-77 (2011). DOI 10.1007/s13389-011-0004-0

17. Skorobogatov, S., Anderson, R.: Optical Fault Induction Attacks. In: B. Kaliski, e. Ko, C. Paar (eds.) Cryptographic Hardware and Embedded Systems - CHES 2002, Lecture Notes in Computer Science, vol. 2523, pp. 2-12. Springer Berlin Heidelberg (2003). DOI 10.1007/3-540-36400-5_2. URL http://dx.doi.org/10. 1007/3-540-36400-5_2

18. Tian, Q., Huss, S.: On Clock Frequency Effects in Side Channel Attacks of Symmetric Block Ciphers. In: New Technologies, Mobility and Security (NTMS), 2012 5th International Conference on, pp. 1 -5 (2012). DOI 10. 1109/NTMS.2012.6208680

19. van Woudenberg, J., Witteman, M., Menarini, F.: Practical Optical Fault Injection on Secure Microcontrollers. In: Fault Diagnosis and Tolerance in Cryptography (FDTC), 2011 Workshop on, pp. 91-99 (2011). DOI 10.1109/ FDTC.2011.12

20. van Woudenberg, J.G.J., Witteman, M.F., Bakker, B.: Improving Differential Power Analysis by Elastic Alignment. In: Proceedings of the 11th International Conference on Topics in Cryptology: CT-RSA 2011, CTRSA'11, pp. 104-119. Springer-Verlag, Berlin, Heidelberg (2011) 
21. Yang, S., Gupta, P., Wolf, M., Serpanos, D., Narayanan, V., Xie, Y.: Power analysis attack resistance engineering by dynamic voltage and frequency scaling. ACM Trans. Embed. Comput. Syst. 11(3), 62:1-62:16 (2012). DOI $10.1145 / 2345770.2345774$

\section{Appendix A: Hardware and Design Details}

This appendix provides some brief notes on the physical hardware realized in this paper, along with a few notes for researchers looking to duplicate it. Note that full details are posted as part of the ChipWhisperer Wiki at http://www. ChipWhisperer.com.

\subsection{Core Clock Recovery Module}

The core part of this work is a module with a Low Noise Amplifier (LNA), Limiter, and Phase-Lock Loop (PLL) chip. The schematic for this is given in Fig. 26. The LNA is an Analog Devices AD8331, which has a variable gain up to $55 \mathrm{~dB}$. A resistor connected to the 'RLIM' pins provides an ability to set an arbitrary clipping level for the output. This clipped output is connected to the PLL chip, which is a Texas Instruments CDCE906. The clipped output from the LNA is used a LVDS input to the PLL, which works assuming the input to the entire block was sufficiently clean, that is to say contains only a single frequency component. Additional filtering can be added by placing capacitors on each of the input pins of the CDCE906 to ground, values between $100 \mathrm{pF}-680 \mathrm{pF}$ are reasonable depending on the fundamental frequency being targeted.

The CDCE906 was chosen for it's ability to operate down to $1 \mathrm{MHz}$, many PLL devices have higher lower frequency limits. If attacking devices with relatively slow internal oscillators, such as the KeeLoq devices at $1.3 \mathrm{MHz}$, this lower range is needed. The CDCE906 can be configured via $\mathrm{I}^{2} \mathrm{C}$ to adjust parameters such as input drive level, frequency divider settings, and outputs in use. For this work it was configured to enable the PLL with frequency dividers such that the input and output frequency were the same. The sampling rate can easily be set to a higher multiple of the system frequency with this PLL block.

\subsection{Filter}

The filter design was done using the Quite Universal Circuit Simulator (QUCS) software. QUCS contains a Filter Synthesis tool, which can be used to generate an appropriate band-pass filter. This will be calculated with 'ideal' component values, and then these values are

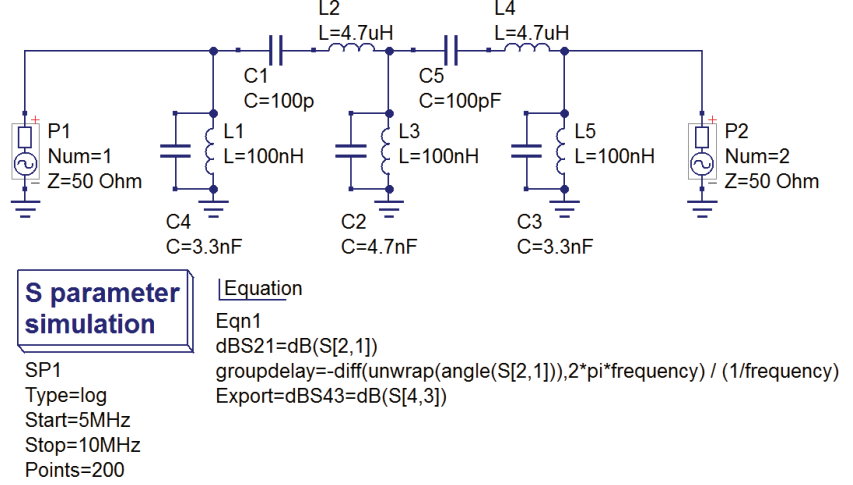

Fig. 27 Bandpass Filter Design Environment. Note the component values have been changed to reflect those being used in the actual circuit, and some optimizations may be needed to get acceptable performance. The equation to plot group delay in clock cycles can be seen in this diagram.

adjusted to the closest standard part, and a simulation confirms if the performance is still acceptable.

Note that at DC the filter will present a dead short, as no blocking capacitors are present. If connecting one side of the filter to a shunt or other device with a DC bias, always insert DC blocking capacitors.

\subsection{First Stage LNA}

An additional LNA may be required in front of the band-pass filter depending on the signal strength. It is possible to use a standard device such as a MiniCircuits $Z F L-1000 L N+$. Care must be taken with RF amplifiers, as most of them are designed for use with $50 \Omega$ systems. If the output or input is not matched properly the amplifier may oscillate, causing errors. Generally amplifiers based on Op-Amps are safer in this regard, and specially-designed differential amplifiers can be exceedingly useful when measuring across current shunts. 
This is the authors version of an article accepted into the Journal of Cryptographic Engineering.

The final publication is available at www.springerlink.com. 10.1007/s13389-014-0087-5

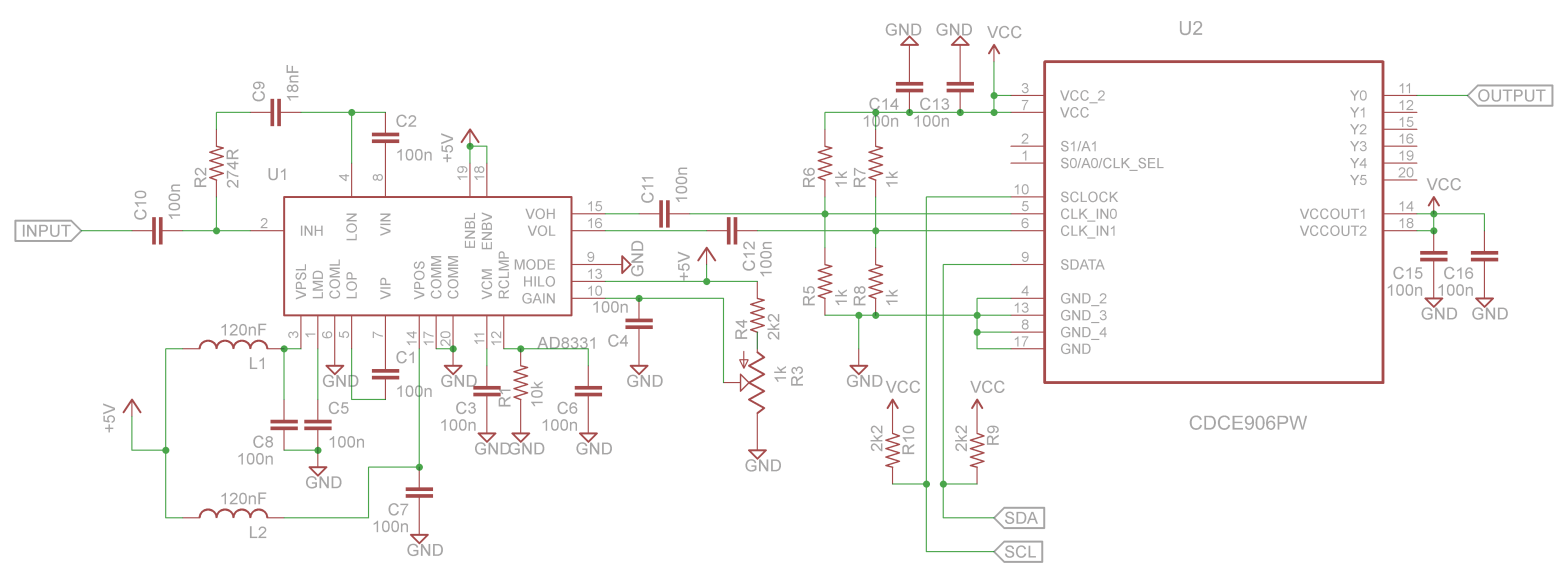

Fig. 26 Schematic for the LNA, Limiter, and PLL as used in Fig. 14. 